\title{
Zoning based Devanagari Character Recognition
}

\author{
O. V. Ramana Murthy \\ Dept. of Electrical Engineering \\ IIT Delhi \\ New Delhi -110016
}

\author{
M. Hanmandlu \\ Dept. of Electrical Engineering \\ IIT Delhi \\ New Delhi -110016
}

\begin{abstract}
In character recognition, zoning based feature extraction is one of the most popular methods. The character image is divided into predefined number of zones and a feature is computed from each of these zones. Usually, this feature is based on the pattern (black) pixels contained in that zone. Some of such features are average pixel density, sum squared distance, histogram. But in such features, say the average pixel density, different combination location of pixels can all give rise to same average pixel density. This often leads to errors in classification.

In this paper, a new technique is presented where the pattern pixel location is also taken into account to contribute as much unique feature as possible. The experimental tests, carried out in the field of Devanagari handwritten numeral and character recognition show that the proposed technique leads to improvement over the traditional zoning methods..
\end{abstract}

\section{General Terms}

Character recognition.

\section{Keywords}

Classification, feature extraction, character recognition, Support Vector Machine.

\section{INTRODUCTION}

Zoning is one of the most popular methods in character recognition for extracting discriminating characteristics. Traditional zoning methods are based on standard partitioning criteria of the character image, as described in the survey papers of Mori et al. [1] and Trier et al. [2]. For instance, various zonings were considered by superimposing a regular grid of sizes $2 \times 2,3 \times 2,1 \times 2$, and $2 \times 1$ on the character image by Suen et al. [3] for recognition of hand-printed characters. Bokser [4] uses a $3 \times 3$ regular grid for zoning design and computes the percentage of black pixels in each zone for classification. Takahashi [5] used vertical, horizontal and diagonal slices as zones. For each zone, the orientation histograms were detected from pattern contours. Several more works [6-10] also used zoning in some form or the other for character recognition.

Zoning has also been used for several works on Indian languages. Negi et al. [11] used a 4x4 grid to derive the density of pixels in the different zones for Telugu script. Hanmandlu and Ramana Murthy [12] used 7x8 grid and derived boxdistance features from each zone for Devanagari script. In [13] grid based method was used to recognize the handwritten Bangla numerals. $4 \times 4$ grid was used in zoning based isolated Kannada numerals recognition by $[14,15]$.

In the features derived from all the above-methods, different combinations of location of pixels can all yield to the same feature value. For example, several combinations of the black pixels can yield to a same average pixel density. This often leads to errors in classification. In this paper, a new technique is presented where the character black pixel location is also taken into account to contribute as much unique feature as possible. Experiments were conducted on Devanagari handwritten numeral and characters using the features with and without the consideration of the black pixel location. SVM is used as the classifier. Results show that the proposed technique leads to improvement over the traditional zoning methods.

The paper is organized as follows. Section II presents the drawback in some features computed from zoning techniques and the proposed improvement. Section III gives a very brief review on SVM used as classifier. Section IV reports the experimental results, carried out on handwritten Devanagari numerals and characters. Contributions of this paper and the conclusions are discussed in Section V.

\section{FEATURE EXTRACTION}

\subsection{Overall system}

The overall recognition approach is briefly described here with a schematic diagram shown in Fig. 1. We extract features using two popular zoning techniques $[4,12]$ from the literature. We then incorporate our proposed improvement in these feature techniques and compare our results with the techniques and results in literature.

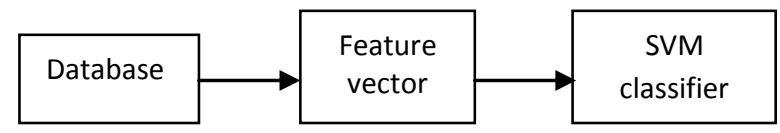

Fig.1. A Schematic diagram of the proposed approach

\subsection{Proposed feature extraction}

In the zoning feature extraction, a character is usually divided into zones of predefined size. These predefined or grid sizes are typically of the order $2 \times 2,4 \times 4$ etc. Some typical zoning of a character is shown in Fig.2.

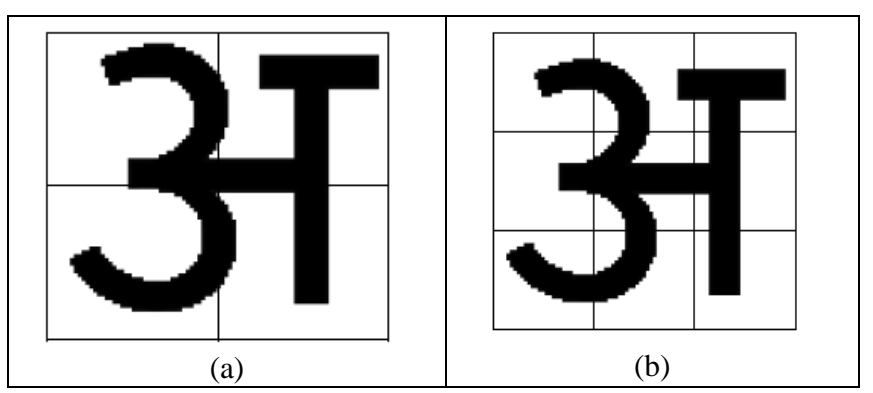




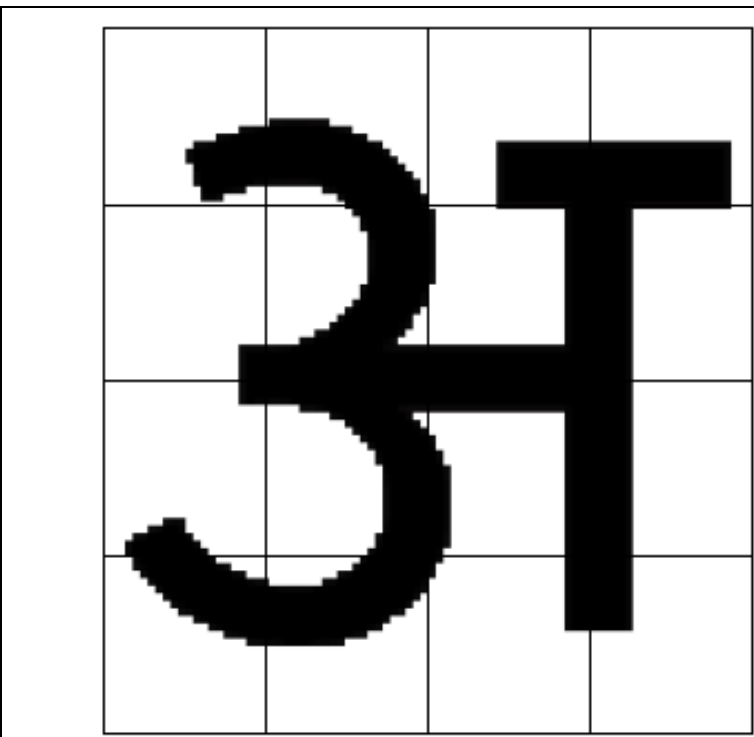

(c)

Fig.2 A character divided into different zones using grid size of (a) $2 \times 2$, (b) $3 \times 3$, (c) $4 \times 4$

While computing the feature from each grid, care has not been taken for each pixel to contribute a unique value. For example, in computing the average pixel density [4], four different combinations of pixels have been shown in Fig. 3 that yields the same average pixel density. Similarly in Fig.4, 4 different combinations have been shown that yield a same box feature value [12]. These errors computed in this feature extraction module creeps in some more error into the system and eventually affecting the recognition performance.

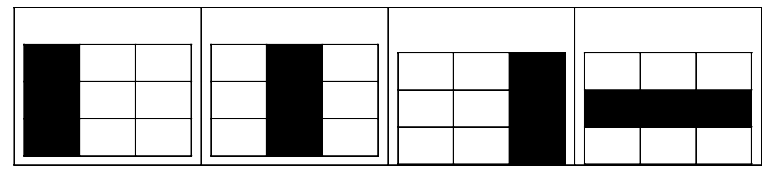

Fig.3 Different combinations of pattern (black) pixels present in a grid of $3 \times 3$ yielding a same average pixel density [4].

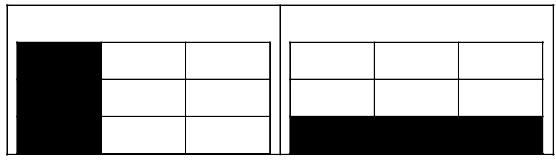

Fig.4 Different combinations of pattern (black) pixels present in a grid of $3 \times 3$ yielding a same Box feature

[12]

The following method is proposed to take care of this drawback in the zoning feature technique.
By considering the bottom left corner of each grid as the absolute origin $(0,0)$, the phase angle of each pixel at $\{x, y\}$ is computed as below

$\theta=\tan ^{-1}\left(\frac{y}{x}\right)$

While computing any feature value [4, 12], each pixel contribution utilizes this value to make it a unique contribution. For instance, the box feature [12] of a grid is computed as follows. By considering the bottom left corner as the absolute origin $(0,0)$, the coordinate distance (Vector Distance) for the $k^{\text {th }}$ pixel in the $b^{\text {th }}$ box at location $(i, j)$ is computed as:

$$
d_{k}^{b}=\left(i^{2}+j^{2}\right)^{\frac{1}{2}}
$$

By dividing the sum of distances of all black pixels present in a box with their total number, a modified box feature is obtained $(\lambda)$ for each box as follows

$\lambda=\frac{1}{N} \sum_{k=1}^{n_{b}} d_{k}^{b}$

where $N$ is total number of pixels in a box. $n_{b}$ is the number of black (pattern) pixels in $b^{\text {th }}$ box.

The modified box feature is computed as follows

$\lambda_{\text {mod }}=\lambda \alpha \cos \theta$

where $\alpha$ is a multiplying factor taken as 1 in our experiments. As the range of $\cos \theta$ is 0 to 1 only, $\alpha$ was introduced.

Similarly, the modified average pixel density is computed as follows.

$\beta=\frac{1}{N} \sum_{k=1}^{n_{b}} \alpha \cos \theta$

\section{CLASSIFIER}

We use Support Vector Machine (SVM) as classifier in this study. The SVM produces a model (based on the training data) which predicts the target values of the test data given only the test data features. An SVM is defined for two-class classification. Given a training set of instance-label pairs $\left(x_{i}, y_{i}\right)$, $i=1,2 \ldots l$ where $x_{i} \in R^{n}$ and $\mathrm{y} \in\{1,-1\}^{l}$, the SVM require the solution of the following optimization problem:

$$
\min _{w, b, \xi} \frac{1}{2} w^{T} w+C \sum_{i=1}^{l} \xi_{i}
$$

Subject to $y_{i}\left(w^{T} \phi\left(x_{i}\right)+b\right) \geq 1-\xi_{i}, \quad \xi_{i}>0$

Here the training vectors $x_{i}$ are mapped into a higher dimensional space by the function $\phi$. SVM finds the optimal hyper-plane which maximizes the distance, or more specifically the margin, between the nearest examples of both the classes. 
These nearest examples are called as support vectors (SVs). C > 0 is the penalty parameter of the error term.

Furthermore, $\quad K\left(x_{i}, x_{j}\right) \equiv \phi\left(x_{i}\right)^{T} \phi\left(x_{j}\right)$ is called the kernel function. We used the radial basis function (RBF) kernel in our work given by $K\left(x_{i}, x_{j}\right) \equiv e^{\left(-\gamma\left\|x_{i}-x_{j}\right\|^{2}\right)}, \gamma>0$.

The SVM is implemented in MatLab [www.mathworks.com] using the LIBSVM [18]. There are two parameters for an RBF kernel: $\mathrm{C}$ and $\gamma$. The parameter search for best $\mathrm{C}$ and $\gamma$ is obtained by conducting a grid search and using cross-validation. Various pairs of $(\mathrm{C}, \gamma)$ values are tried and the one with the best cross-validation accuracy is picked. Trying exponentially growing sequences of $\mathrm{C}$ and $\gamma$ is a practical method to identify good parameters (for $\mathrm{C}=2^{-5}, 2^{-3}, \ldots, 2^{15}$, and $\gamma=2^{-3}, 2^{-1}, \ldots$, $2^{15}$ ).

\section{RESULTS AND DISCUSSION}

The proposed feature extraction technique is applied on Devanagari numeral and character databases. The details of the two databases are given in Table 1. A snapshot of each database is shown in Fig.5 and Fig. 6 below. The recognition rates are summarized in Tables 2-5.

Table 1: Details of Database used

\begin{tabular}{|l|l|l|l|}
\hline Database & Source & $\begin{array}{l}\text { No of } \\
\text { classes }\end{array}$ & $\begin{array}{l}\text { Size of } \\
\text { database }\end{array}$ \\
\hline Numerals & {$[12]$} & 10 & 3500 \\
\hline characters & {$[17]$} & 36 & 4713 \\
\hline
\end{tabular}

For each database, character is preprocessed and normalized to a size of $32 \times 32$ [3]. Grid sizes of $2 \times 2,4 \times 4,8 \times 8,16 \times 16$ were used for zoning and extracting the features. Thus, the influence of grid size was also studied.

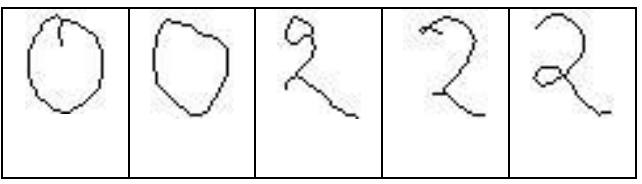

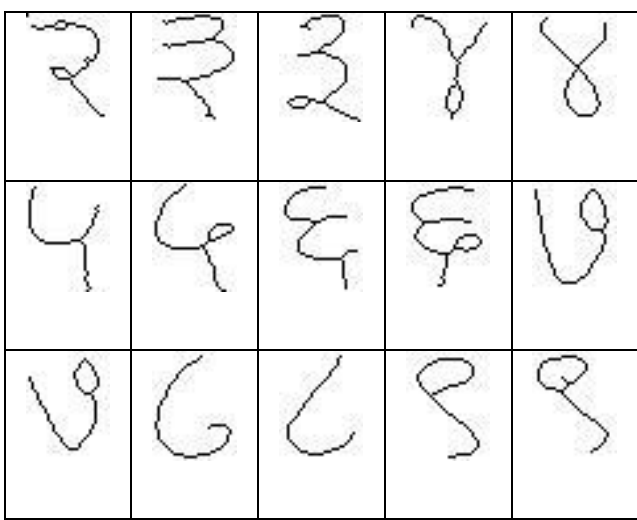

Fig. 5: Snap shot of preprocessed Devanagari Numerals (courtesy [12])

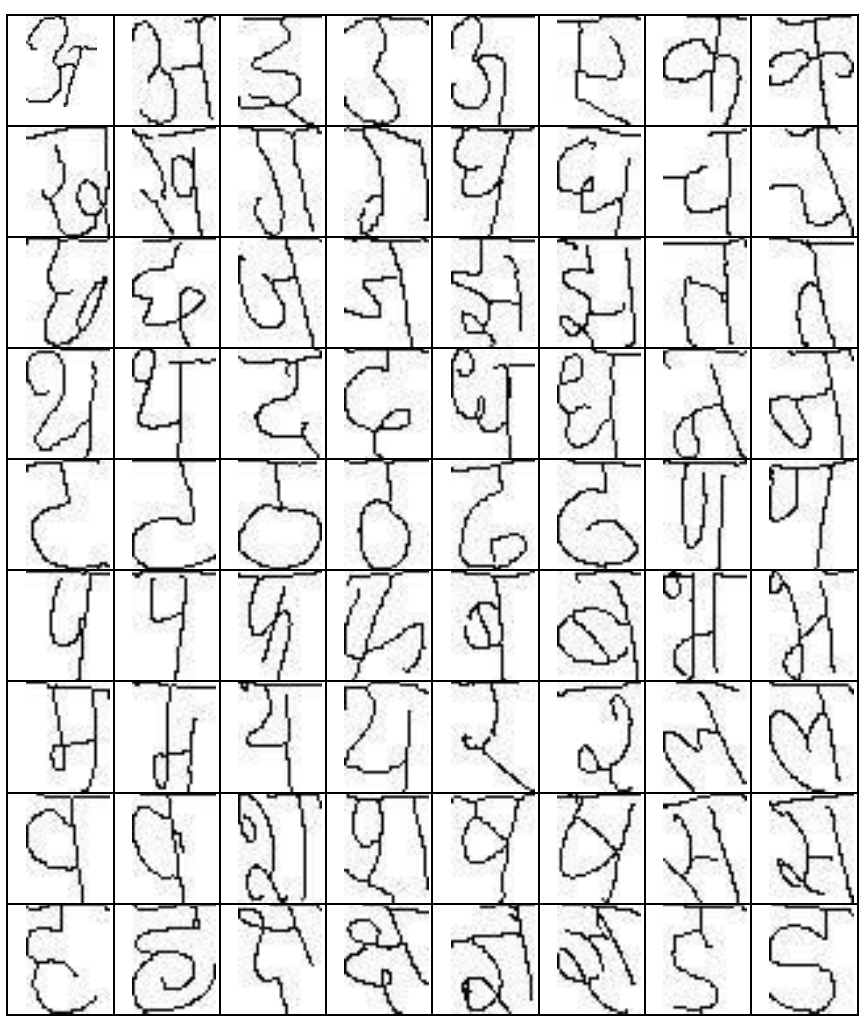

Fig. 6: Snap shot of preprocessed Devanagari Characters (courtesy [17])

Table 2: Results of Box features on Devanagari Numerals using Box features [12]

\begin{tabular}{|c|c|c|c|c|c|c|c|}
\hline \multirow{2}{*}{$\begin{array}{c}\text { Size of } \\
\text { grid }\end{array}$} & $\begin{array}{c}\text { No. of } \\
\text { features }\end{array}$ & \multicolumn{2}{|c|}{ Direct method } & \multicolumn{3}{c|}{ Proposed method } \\
\cline { 3 - 8 } & & Recognition rate & $\mathrm{C}$ & $\gamma$ & $\begin{array}{c}\text { Recognition } \\
\text { rate }\end{array}$ & $\mathrm{C}$ & $\gamma$ \\
\hline $2 \times 2$ & 256 & 90.6 & 1 & -2 & 99.5 & 3 & -6 \\
\hline $4 \times 4$ & 64 & 99.5 & 0 & -2 & 99.6 & 1 & -3 \\
\hline $8 \times 8$ & 16 & 98.9 & 3 & -1 & 98.9 & 3 & -1 \\
\hline $16 \times 16$ & 4 & 90.0 & 12 & 1 & 92.6 & 7 & 9 \\
\hline
\end{tabular}

Table 3: Results of Box features on Devanagari Numerals using pixel density[4] 


\begin{tabular}{|c|l|c|c|c|c|c|}
\hline \multirow{2}{*}{ Size of grid } & \multicolumn{3}{|c|}{ Direct method } & \multicolumn{3}{c|}{ Proposed method } \\
\cline { 2 - 7 } & Recognition rate & $\mathrm{C}$ & $\gamma$ & $\begin{array}{c}\text { Recognition } \\
\text { rate }\end{array}$ & $\mathrm{C}$ & $\gamma$ \\
\hline $2 \times 2$ & 99.4 & $1,-2$ & & 99.4 & 1 & -3 \\
\hline $4 \times 4$ & 99.6 & 0,0 & & 99.6 & 0 & 1 \\
\hline $8 \times 8$ & 98.9 & 3,4 & & 99.1 & 3 & 3 \\
\hline $16 \times 16$ & 92.5 & 4,12 & & 91.3 & 10 & 11 \\
\hline
\end{tabular}

Table 4: Results of Box features on Devanagari characters using Box Features [12]

\begin{tabular}{|c|l|c|c|c|c|c|}
\hline \multirow{2}{*}{ Size of grid } & \multicolumn{3}{|c|}{ Direct method } & \multicolumn{3}{c|}{ Proposed method } \\
\cline { 2 - 7 } & Recognition rate & $\mathrm{C}$ & $\gamma$ & $\begin{array}{c}\text { Recognition } \\
\text { rate }\end{array}$ & $\mathrm{C}$ & $\gamma$ \\
\hline $2 \times 2$ & 37.8 & 2 & -2 & 68.2 & 4 & -4 \\
\hline $4 \times 4$ & 85.7 & 1 & -2 & 88.6 & 2 & -4 \\
\hline $8 \times 8$ & 78.9 & 2 & -1 & 80.0 & 3 & -2 \\
\hline $16 \times 16$ & 39.6 & 7 & 3 & 41.1 & 9 & 1 \\
\hline
\end{tabular}

Table 5: Results of Box features on Devanagari characters using pixel density [4]

\begin{tabular}{|c|l|c|c|c|c|c|}
\hline \multirow{2}{*}{ Size of grid } & \multicolumn{3}{|c|}{ Direct method } & \multicolumn{3}{c|}{ Proposed method } \\
\cline { 2 - 7 } & Recognition rate & $\mathrm{C}$ & $\gamma$ & $\begin{array}{c}\text { Recognition } \\
\text { rate }\end{array}$ & $\mathrm{C}$ & $\gamma$ \\
\hline $2 \times 2$ & 82.4 & 2 & -2 & 84.6 & 2 & -4 \\
\hline $4 \times 4$ & 88.9 & 2 & 0 & 88.1 & 2 & -1 \\
\hline $8 \times 8$ & 80.7 & 7 & 5 & 79.8 & 2 & 4 \\
\hline $16 \times 16$ & 34.2 & 10 & 5 & 40.3 & 13 & 7 \\
\hline
\end{tabular}

\subsection{Discussion}

Handwritten Devanagari numeral recognition results are summarized in Table 2 and 3. There is significant improvement when the grid $2 \times 2$ was used for zoning. But when the grids $4 \times 4$, $8 \times 8$ and $16 \times 16$ were used, the performance was nearly equal and one time lesser than the original feature value. This is due to the range of $\cos \theta$ being 0 to 1 only. Hence choice of $\alpha$ will be taken up in future to study it's effect. Also as the performances are nearly above $98.5 \%$, a slight difference in decimal places can be discounted. Overall, it is significant to see that a grid size of $4 \times 4$ yields best results, as supported by literature [11].

Handwritten Devanagari character recognition results are shown in Tables 4 and 5. As the number of classes is 36, greater than that in the numerals, there is considerable decrease in the overall performance decline from $98 \%$ to $85 \%$. Certainly this calls for design of features and classifiers, collection of more samples etc. In this experiment, the study of zoning features revealed that there is significant improvement in the recognition rate for any grid size. A comparison of our work with literature is summarized in Table 6 .

Table 6: Comparison with other works

\begin{tabular}{|c|c|c|c|c|}
\hline Database & Reference & $\begin{array}{c}\text { Length } \\
\text { of } \\
\text { features }\end{array}$ & Classifier & $\begin{array}{c}\text { Recognition } \\
\text { rate }\end{array}$ \\
\hline $\begin{array}{c}\text { ISI(Datasize } \\
\text { 11270) }\end{array}$ & {$[19]$} & 64 & SVM & 80.36 \\
\hline $\begin{array}{c}\text { IIT(Datasize } \\
\text { 4750) }\end{array}$ & $\begin{array}{c}\text { Our } \\
\text { proposed } \\
\text { features }\end{array}$ & 64 & SVM & 88.9 \\
\hline
\end{tabular}

Training of SVM takes a lot of time and memory also. Several hours were spent to find the design parameters $C$ and $\gamma$ for each case. Study of other classifiers is also required. Also the range of $\cos \theta$ is 0 to 1 only. Hence a choice of proper multiplying factor $\alpha$ is required.

\section{CONCLUSIONS AND FUTURE WORK}

Zoning is one of the popular techniques for feature extraction in character recognition. But it has a drawback that different combinations of pattern pixels can yield to same feature value. A method has been proposed to modify such feature values computed from the zones so that each pattern pixel contributes a unique value to feature computation. By this modified feature value, improvement or nearly equal performance has been observed in the recognition rates of Devanagari handwritten numerals and characters.

Several other works in literature $[19,20]$ have also utilized the phase information in their experiments. But it was used separately along with gradient etc information. The contribution of this paper is in it's utility of phase information for exclusive unique contribution of each pixel value in a given zone.

\section{REFERENCES}

[1] S. Mori, C. Y. Suen, and K. Yamamoto, "Historical review of OCR research and development," Proc. IEEE, vol. 80, no. 7, pp. 1029-1058, Jul. 1992.

[2] O. D. Trier, A. K. Jain, and T. Taxt, "Feature extraction methods for character recognition-A survey," Pattern Recognition, vol. 29, no. 4, pp. 641-662, 1996.

[3] C. Y. Suen, J. Guo, and Z. C. Li, "Analysis and recognition of alphanumeric handprints by parts," IEEE Trans. Syst., Man, Cybern., vol. 24, no. 4, pp. 614-630, Apr. 1994. 
[4] M. Bokser, "Omnidocument technologies," Proc. IEEE, vol. 80, no. 7, pp. 1066-1078, Jul. 1992.

[5] H. Takahashi, "A neural net OCR using geometrical and zonal pattern features," in Proc. Int. Conf. Document Analysis and Recognition, Saint-Malo, France, 1991, pp. 821-828.

[6] G. Baptista and K. M. Kulkarni, "A high accuracy algorithm for recognition of hand-written numerals," Pattern Recognition, vol. 21, no. 4, pp. 287-291, 1988.

[7] G. Dimauro, S. Impedovo, R. Modugno, and G. Pirlo, "Numeral recognition by weighting local decisions," in Proc. Int. Conf. Document Analysis and Recognition, Edinburgh, U.K., Aug. 2003, pp. 1070-1074.

[8] F. Kimura and M. Shridhar, "Handwritten numerical recognition based on multiple algorithms," Pattern Recognition, vol. 24, no. 10, pp. 969-983, 1991.

[9] S.-H. Cha, C. C. Tappert, and S. N. Srihari, "Optimizing binary feature vector similarity using genetic algorithm and handwritten character recognition," in Proc. Int. Conf. Document Analysis and Recognition, Edinburgh, U.K., Aug. 2003, pp. 662-665.

[10] J. Cao, M. Ahmadi, and M. Shridhar, "Handwritten numeral recognition with multiple features and multistage classifiers," in IEEE Int. Symp. Circuits and Systems, London, U.K., 1994, pp. 323-326.

[11] A. Negi, K. N. Shanker, and C. K. Chereddi, "Localization, extraction and recognition of text in Telugu document images," in Proc. Int. Conf. Document Analysis and Recognition, Edinburgh, U.K., Aug. 2003, pp. 1193-1197.

[12] M. Hanmandlu and O. V. Ramana Murthy "Fuzzy model based recognition of handwritten numerals", Pattern Recognition, 40 (6), (2007), 1840-1854.
[13] A. Majumdar and B.B. Chaudhuri, "Printed and handwritten numeral recognition using multiple classifier outputs", proceedings of the first IEEE ICSIP06, Vol.1, pp.190-195, 2006

[14] G.G.Rajaput and Mallikarjun Hangarge, "Recognition of isolated handwritten Kannada numerals based on image fusion method", PReMI07, LNCS 4815, pp.153-160, 2007

[15] S.V.Rajashekaradhya and P.Vanaja Ranjan, "Isolated handwritten Kannada digit recognition: A novel approach", proceedings of the International conference on Cognition and Recognition”, pp.134-140, 2008

[16] V. Vapnik, "The Nature of Statistical Learning Theory", Springer Verlang, 1995.

[17] M. Hanmandlu, O. V. Ramana Murthy, Vamsi Krishna Madasu, "Fuzzy Model based recognition of handwritten Hindi characters", Digital Image Computing Techniques and Applications, DICTA 3-5 Dec 2007, pp. 454-461.

[18] Chih-Chung Chang and Chih-Jen Lin, " LIBSVM a library for support vector machines", ACM Transactions on Intelligent Systems and Technology, 2:27:1--27:27, 2011.

[19] N. Sharma, U. Pal, F. Kimura and S. Pal, "Recognition of Offline Handwritten Devnagari Characters using Quadratic Classifier", In Proc. Indian Conference on Computer Vision Graphics and Image Processing, pp- 805-816, 2006.

[20] U. Pal, T. Wakabayashi and F. Kimura, "Comparative Study of Devnagari Handwritten Character Recognition using Different Feature and Classifiers", In Proc. 10th International Conference on Document Analysis and Recognition (ICDAR), pp.1111-1115, 2009. 